Knox, R., Swait, E. \& Woodroffe, R. (1956). J. gen. Microbiol. 15, 359-371

\title{
The Growth of Mycobacterium tuberculosis in Semi-solid Agar Media
}

\author{
BY R. KNOX, EILEEN SWAIT AND R. WOODROFFE \\ Department of Bacteriology, Guy's Hospital Medical School, London, S.E. 1
}

\begin{abstract}
SUMMARY: Further experience of semi-solid agar media has fully confirmed the earlier opinion (Knox, 1955a) of their great value for culture of Mycobacterium tuberculosis. The media mainly used were those of Kirchner, Fisher and Dubos, made semi-solid with $0.125 \%$ agar and containing serum or bovine albumin. Large inocula of M. tuberculosis $\mathrm{H} 37 \mathrm{Rv}$ strain in Kirchner semi-solid agar gave easily visible growth in 2-3 days when the simple method of viewing described was used; small inocula gave countable colonies in 10-14 days, or in 7-10 days when a hand-lens was used. Maximum or nearly maximum viable counts were reached in about 2 weeks, and the final number of viable organisms recovered was as high as or higher than in any other media used. The medium also gave rapid growth of other strains of $M$. tuberculosis including drug-resistant variants of $\mathrm{H} 37 \mathrm{Rv}$, strains of $M$. tuberculosis var. bovis, and of B.C.G. and strains isolated from patients' sputa. Semi-solid media are easy to prepare and safe to handle. Contamination rates are low, evaporation does not occur when rubber stoppers are used, and large numbers of cultures can be inoculated and incubated in a small space. Repeated readings can be made without opening the culture tubes. Viable counts and drug sensitivity tests are easy to read and record. For drug sensitivity tests semi-solid media, besides giving, when serial drug dilutions are used, an initial end-point which is as easy to read as with liquid media, also give information not given by liquid media as to the proportion of 'resistant' organisms present in a given culture. Semi-solid agar media are of great value for any work involving speed in culture, drug sensitivity tests or viable counts of M. tuberculosis.
\end{abstract}

In an earlier paper (Knox, 1955a) it was shown that semi-solid agar media were of great value for many different types of work requiring rapid and reliable growth of tubercle bacilli. The purpose of the present paper is to give in greater detail the method used and to describe further experiments which have confirmed and extended the original findings.

\section{METHODS}

Organisms. The organisms mainly used were the normal drug-sensitive laboratory strain of Mycobacterium tuberculosis var. hominis $\mathrm{H} 37 \mathrm{Rv}$ and a laboratory trained variant ( $\mathrm{R} 1$ ) made resistant to $10 \mu \mathrm{g}$. isoniazid $/ \mathrm{ml}$. in Dubos liquid medium (Knox, $1955 \mathrm{~b}$ ). In a few experiments other strains were used. These included two other laboratory trained resistant variants of the $\mathbf{H} \mathbf{3 7} \mathbf{R v}$ strain : R 3, resistant to $200 \mu \mathrm{g}$. streptomycin $/ \mathrm{ml}$. and R 4 , resistant to $10 \mu \mathrm{g}$. isoniazid $/ \mathrm{ml}$. and $200 \mu \mathrm{g}$. streptomycin $/ \mathrm{ml}$; ; a bovine strain of $\boldsymbol{M}$. tuberculosis obtained from Professor Robson; a drug-sensitive strain of B.C.G. and a variant of it resistant to $10 \mu \mathrm{g}$. isoniazid $/ \mathrm{ml}$.; several strains of $M$. tuberculosis var. hominis isolated from patients' sputa. These cultures were maintained by 
subculture, usually every 3 months, on Lowenstein Jensen medium. Subcultures were made in Dubos \& Davis (1946) liquid medium for inoculation into other media.

Media. Liquid media used were: Kirchner medium prepared as described by Mackie \& McCartney (1953); Fisher medium (Fisher, 1952); Dubos medium (Dubos \& Davis, 1946). The first two media were made in this laboratory, the last was supplied by the Southern Group Laboratory (Park Hospital, Hither Green, London, S.E. 13). Semi-solid media were made by adding to these liquid media agar in a final concentration of $0.125 \mathrm{~g} .100 \mathrm{ml}$., except in experiments in which the agar concentration was deliberately varied. Horse serum (supplied by Burroughs Wellcome and Co.) sterilized by Seitz filtration was added aseptically to Kirchner or Fisher medium to give a final concentration of $10 \%$, and bovine serum albumin fraction V $(0.35 \%)$ to Dubos medium which already contained Tween $80(0.05 \%)$. For use in experiments, the semisolid agar media were heated to $100^{\circ}$ to melt the agar and cooled in a water bath at $48^{\circ}$. Horse serum or bovine serum albumin was added at this temperature immediately before the medium was distributed into sterile $6 \times \frac{5}{8}$ in. test tubes plugged with cotton wool and kept in racks in a water bath at $48^{\circ}$ ready for inoculation. Other media used were Kirchner, Fisher, and Dubos media with $1.5 \%$ agar, Hirsch's charcoal medium with $1 \%$ agar (Hirsch, 1954) and glycerol blood agar with $50 \%$ blood similar to the medium described by Tarshis (1953). These media (except for the liquid Dubos base) were made in the laboratory and distributed for use in Petri dishes. In addition, Lowenstein Jensen slopes in screw-capped bottles were supplied by the Southern Group Laboratory.

Method of inoculation. Cultures of Mycobacterium tuberculosis in Dubos liquid medium were diluted, usually in tenfold steps, in distilled water unless otherwise stated. Inoculation was by means of dropping pipettes calibrated to deliver $0.02 \mathrm{ml}$./drop. Each tube containing semi-solid agar was removed from the $48^{\circ}$ bath, inoculated and placed in a rack on the bench ready for incubation at $37^{\circ}$. Usually before incubation, or sometimes after overnight incubation, cotton-wool plugs were removed and replaced by rubber bungs or the rubber seals made for viable counts in roll tubes by Astell Laboratory Service Co. Ltd., 172 Brownhill Road, London, S.E. 6. These prevented evaporation as efficiently as rubber bungs and were considerably easier to handle in large numbers. Cultures were incubated at $37^{\circ}$ in air.

Examination of cultures. Inoculated tubes were examined after 2-3 days and at intervals afterwards. Early growth was made more easily visible by placing each tube to be examined in xylol or ethanol in a beaker or similar container. This had the effect of cleaning the outsides of the culture tubes and eliminated the optical effects of scratch-marks and troublesome reflexions from the walls of the tubes (Knox, 1955a). A special viewing-box was made, suitable for routine observation of large numbers of tubes. It had two compartments, an upper in which the xylol-containing beaker was suspended, and a lower containing an electric light bulb which threw its light through the bottom of the beaker obliquely upwards on to the culture tubes to be examined. 
The relative position of the beaker and of the light source could be adjusted, so as to give the best oblique illumination for viewing. Cultures were viewed either with a rectangular lens supplied by Britex (Scientific Instruments) Ltd. 329 High Holborn, W.C. 1, giving a magnification of about $\times 2$, or with a hand-lens $(\times 6)$, or by naked eye. Cultures were usually examined at intervals up to 3 or 4 weeks, but for special purposes longer periods of incubation were used. For microscopic examination, colonies in semi-solid agar were removed with a Pasteur pipette and a drop was placed on a glass slide. A strong straight wire was used to break down the colonies as much as possible and to remove lumps of agar. After drying and fixing by slight heating a Ziehl-Neelsen stain was made in the usual fashion.

\section{RESULTS}

Growth of Mycobacterium tuberculosis $H 37 R v$ in Kirchner's medium with $0 \cdot 125 \%$ agar

The standard medium. The 'standard' medium for most of the experimental work was Kirchner's medium with $0.125 \%$ agar distributed in $2 \mathrm{ml}$. amounts in 6 in. $\times \frac{5}{8}$ in. tubes with rubber stoppers to prevent evaporation. The growth of Mycobacterium tuberculosis $\mathbf{H ~} 37 \mathrm{Rv}$ at $37^{\circ}$ in this medium will therefore be described in some detail. The appearances described are those which were seen with standard conditions of lighting (see Methods).

When a large inoculum was used $(0.04 \mathrm{ml}$. of a 10 to 14-day culture of the $\mathrm{H} 37 \mathrm{Rv}$ strain in Dubos medium undiluted or diluted 1/10), growth in Kirchner semi-solid agar medium was first visible in 2-3 days. To the naked eye it appeared as a cloudiness of the medium but with a hand-lens $(\times 4$ or $\times 6$ ) uncountable numbers of minute colonies could be seen. By the end of 4-5 days, towards the bottom of the tubes the growth still appeared as uncountable numbers of minute colonies now easily visible with the naked eye, but near, and usually $2-3 \mathrm{~mm}$. below, the surface of the agar the individual colonies had coalesced so as to form a band of confluent growth. Growth in this zone continued rapidly. At 10-14 days surface growth began to appear in the form of a pellicle creeping up the sides of the tube, but the minute colonies in the lower part of the medium showed no increase in size. When smaller inocula were used, the appearances were different. With inocula of the order of 100-1000 organisms, minute colonies were first visible at about 5-6 days. On further incubation these increased in size throughout the medium at first, but by 7-10 days the colonies at, or just below, the surface were already becoming larger than the colonies lower down in the agar, and this process continued on further incubation. With inocula of $\mathbf{5 0}$ or fewer organisms, as for example with a $10^{-5}$ or $10^{-6}$ dilution of a Dubos culture, colonies were first visible to the naked eye at about 7-10 days, though clearly visible with a hand-lens at 4-6 days. On further incubation they continued to increase in size, but not greatly in number, and at 10-14 days they were usually of fairly uniform size throughout the medium. On continued incubation, however, the colonies near the surface grew somewhat larger than those lower down. When, however, the 
number of colonies was as small as 10-20 they usually remained fairly uniform in size up to 2-3 weeks, by which time they were 1-1 $\frac{1}{2} \mathrm{~mm}$. in diameter. Single colonies in Kirchner medium were well defined and roughly spherical in shape, but their surfaces were irregular. Some typical cultures are illustrated in Pl. 1, fig. 1. On prolonged incubation, for 3-4 weeks and longer, secondary colonies began to appear in a track extending vertically downwards from the parent colony (Pl. 1, fig. 2). Ziehl-Neelsen stained films of colonies showed typical acid-ethanol fast bacilli, but no attempt has yet been made to see whether the individual organisms in colonies of different age or at different depths in the agar show any differences in morphology.

Many experiments were performed to investigate the effect of variations in the composition and distribution of the medium, and of changes in the conditions of incubation. These experiments were too numerous to give in full, but the main results will be described.

\section{Variations in composition of the medium}

Type of agar. In a few experiments Davis agar supplied by Davis Gelatine (NZ) Ltd., Christchurch, New Zealand, was compared with Oxoid agar (Oxo Ltd., Thames House, Queen Street Place, E.C. 4). In general, growth appeared more rapidly and colonies were larger with Davis agar. In some experiments the number of viable organisms was greater in this agar. Since this gave satisfactory results, the same batch of this was used throughout and no further comparisons were made.

Concentration of agar. In liquid Kirchner medium growth of the $\mathrm{H} 37 \mathrm{Rv}$ strain was first visible in 3-5 days in the form of floccules whose size and rate of growth varied with the size of the inoculum. On the addition of agar, even in as low a concentration as $0.01 \%$, growth appeared at first in the form of discrete colonies, the number varying with the concentration. These at first remained suspended in the medium but later sank to the bottom of the tube. The gel formed at this concentration of agar was very weak, and in the presence of serum it sometimes contracted into a compact ball containing most of the inoculated organisms. At concentrations of agar between 0.05 and $0.2 \%$ the gel was fairly firm, and colonies remained separate for 2-3 weeks at $37^{\circ}$. At higher agar concentrations the medium was more opaque, growth was much more difficult to recognize and slower in appearing, separate colonies were smaller and viable counts were considerably lower: The optimum agar concentration was $0 \cdot 1-0 \cdot 125 \%$. A typical experiment is illustrated in Table 1.

The basal medium. In a number of experiments semi-solid agar medium was prepared with basal media other than Kirchner medium; usually Fisher (Fisher, 1952) and Dubos (Dubos \& Davis, 1946) media were tested. The first two media contained $10 \%$ horse serum and the third $0.35 \%$ bovine serum albumin fraction V and $0.05 \%$ Tween 80 . Growth of Mycobacterium tuberculosis in semi-solid Kirchner or Fisher media was very similar, but quite different in semi-solid Dubos agar. With large inocula, growth in Dubos agar was more diffuse than in the other two media and closely resembled the growth in liquid Dubos medium examined after thorough shaking of the sedimented organisms. 
With smaller inocula, colonies appeared in the form of vertical streaks in the Dubos medium and they seldom showed the irregular spherical shape characteristic of their growth in Kirchner semi-solid medium (Pl. 1, fig. 3). Comparisons of the viable counts obtained with these and other media are given in a later section.

Table 1. Effect of agar concentration on viable-counts of Mycobacterium tuberculosis $H 37 R v$

Medium: Kirchner medium with $10 \%$ horse serum. Readings after 21 days of incubation at $\mathbf{3 7}{ }^{\circ}$.

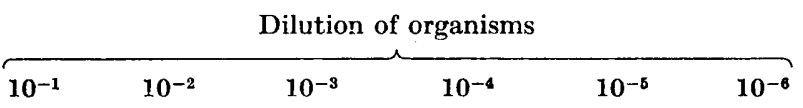

Agar concentration

Relative amount of growth (colonies)

0
$0 \cdot 01$
$0 \cdot 05$
$0 \cdot 125$
$0 \cdot 2$
$0 \cdot 4 *$ (rather opaque)
$0 \cdot 8 *$ (opaque)
$1 \cdot 6 *$ (very opaque)

\begin{tabular}{cccccc}
\multicolumn{5}{c}{ Relative amount of growth (colonies) } \\
++ & ++ & + & + & tr & 0 \\
C & C & SC & unc & 14 & 2 \\
C & C & SC & unc & 18 & 2 \\
C & C & SC & unc & 25 & 2 \\
C & C & SC & unc & 15 & 2 \\
C & C & SC & unc & 15 & 0 \\
C & C & SC & unc & 10 & 4 \\
& & & & (small) & (small) \\
C & C & SC & few & 5 & 0
\end{tabular}

$\mathrm{C}=$ confluent $; \mathrm{SC}=$ semi-confluent $;$ unc $=$ uncountable $; *$ = growth in these concentrations of agar was mainly at top of medium.

The effect of serum. In Kirchner's medium Mycobacterium tuberculosis $\mathrm{H} 37 \mathrm{Rv}$ grew well only in the presence of serum. Growth even of large inocula was erratic without serum or with concentrations of serum up to $0.1 \%$; at concentrations of serum from 0.5 to $10 \%$ growth was rapid and viable counts were satisfactory. Table 2 shows a typical experiment.

Distribution of the medium. Many experiments were performed to find the best type of container and the most satisfactory volume and depth of agar. Too deep a column of agar was unsatisfactory since even with a small inoculum (20-40 organisms) colonies at the bottom remained minute while colonies at the surface grew large. On the other hand, too short a column of agar was also unsatisfactory. With equal inocula, viable counts were higher and colonies were larger and more uniform when the medium was $2-3 \mathrm{~cm}$. deep than when it was only $1 \mathrm{~cm}$. deep or less. From these experiments it was found that the best results were obtained by distributing the medium in volumes of $2 \mathrm{ml}$., or not more than $4 \mathrm{ml}$, in 6 in. $\times \frac{5}{8}$ in. tubes and incubating them with rubber stoppers.

The temperature of the medium at the time of inoculation was important. When the medium was allowed to cool before inoculation the agar, though it never became solid, became too viscous to allow even dispersal of the organisms. When inoculated tubes of semi-solid Kirchner medium were held in the water bath at $48^{\circ}$ for $1 \mathrm{hr}$. after inoculation there was no decrease in the viable 
count. A considerable decrease in viable count, however, occurred when tubes were inoculated first and kept at $48^{\circ}$ for $1 \mathrm{hr}$. before the addition of the medium. The most satisfactory results were given when the semi-solid medium after distribution into test tubes was held at $48^{\circ}$ before inoculation and each tube immediately after inoculation was removed to room temperature ready for subsequent incubation at $37^{\circ}$. This procedure was therefore adopted as a routine.

Table 2. Effect of serum concentration on viable counts of Mycobacterium tuberculosis $\mathrm{H} \mathbf{3 7} \mathrm{Rv}$

Medium: Kirchner with $0.125 \%$ agar. Inoculum $0.04 \mathrm{ml}$. Readings after 14 days of incubation at $37^{\circ}$.

\begin{tabular}{|c|c|c|c|c|c|c|}
\hline \multirow{3}{*}{$\begin{array}{c}\text { Serum } \\
\text { concentration } \\
(\%)\end{array}$} & \multicolumn{6}{|c|}{ Dilution of organisms } \\
\hline & $10^{-1}$ & $10^{-2}$ & $10^{-3}$ & $10^{-4}$ & $10^{-5}$ & $10^{-8}$ \\
\hline & \multicolumn{6}{|c|}{ Colonies counted or otherwise (see key) } \\
\hline & 0 & 0 & 0 & 0 & 0 & 0 \\
\hline 0.01 & SC & o & $\mathbf{0}$ & $\mathbf{0}$ & $\mathbf{0}$ & $\mathbf{0}$ \\
\hline $0 \cdot 05$ & $\mathbf{0}$ & $\mathbf{0}$ & $\mathbf{0}$ & $\mathbf{0}$ & $\mathbf{0}$ & $\mathbf{0}$ \\
\hline $0 \cdot \mathbf{I}$ & 0 & 0 & 0 & 0 & 0 & 0 \\
\hline 0.5 & $\mathbf{C}$ & SC & une & 10 & 2 & $\mathbf{0}$ \\
\hline $1 \cdot 0$ & C & SC & une & 38 & 4 & $\mathbf{0}$ \\
\hline $\mathbf{2 \cdot 0}$ & C & SC & unc & 54 & 6 & $\mathbf{0}$ \\
\hline $3 \cdot 0$ & $\mathbf{C}$ & SC & unc & - & 9 & 1 \\
\hline $4 \cdot 0$ & $\mathbf{C}$ & SC & unc & une & 12 & 1 \\
\hline $5 \cdot 0$ & C & SC & unc & une & 9 & 1 \\
\hline $7 \cdot 5$ & $\mathbf{C}$ & SC & unc & unc & 8 & 1 \\
\hline $10 \cdot 0$ & C & SC & unc & une & 7 & 1 \\
\hline
\end{tabular}

Conditions of incubation. In early experiments tubes of semi-solid agar after inoculation were incubated in air at $37^{\circ}$ with cotton-wool plugs. It was soon clear that the medium became concentrated by evaporation and increasingly inhibitory to continued growth. With rubber stoppers evaporation was negligible, the medium remained semi-solid indefinitely at $37^{\circ}$, and separate colonies continued to increase in size. At any rate with small inocula, growth was as good in tubes incubated with rubber stoppers for 4 weeks as in tubes which were opened to the air by removing the stoppers once a week and replacing them.

In several experiments parallel sets of inoculated tubes were incubated at $37^{\circ}$ with rubber stoppers in an incubator and with cotton-wool plugs in a tin containing air but with only a small outlet to the air of the incubator. No appreciable evaporation occurred in either set of tubes, but in the tubes with the rubber stoppers growth was more rapid and separate colonies were larger than in the tubes in the tin. Further experiments are in progress on the effect of variations in the atmosphere to which the organisms are exposed. 
Viable counts of Mycobacterium tuberculosis $H \mathbf{3 7} R \boldsymbol{v}$

The main characters of the growth of Mycobacterium tuberculosis $\mathbf{H} 37 \mathbf{R v}$ in Kirchner semi-solid agar have already been described. In many experiments designed for different purposes a viable count of the culture was included. For various reasons, results were read after different periods of incubation-in some experiments after 1, 2, 3 and more weeks of incubation, in others at irregular intervals. Each observation was recorded under the appropriate day of incubation together with the final viable count for the same tube. Fortysix experiments are available for analysis. A table was thus constructed showing the average viable counts obtained on different days of incubation and expressed as a proportion of the final viable count. The average number of colonies in $0.04 \mathrm{ml}$. of a $10^{-6}$ dilution of a Dubos culture was about 10; the average viable count was therefore about $250 \times 10^{6}$ organisms $/ \mathrm{ml}$. Up to the ninth day only a small proportion of the colonies finally countable were easily visible to the naked eye, though a much higher proportion could be counted even as early as the seventh day when a hand-lens was used. On the tenth day and after, the number of colonies visible to the naked eye rose sharply and by the end of the second week approached the $100 \%$ level (Table 3). Incubation for 2 weeks is therefore a safe routine for accurate viable counts, though for special purposes or where speed is important, a rough estimate of the number of viable organisms can be given in 7-10 days. Viable counts after more than 4 weeks of incubation were not usually reliable because of the appearance of secondary colonies. Some typical viable counts are shown in Pl. 1.

Table 3. Viable counts after different periods of incubation expressed as $\%$ of the final viable count

$\begin{array}{cc}\text { Naked eye counts of colonies recorded. } \\ \begin{array}{c}\text { Incubation } \\ \text { period }\end{array} & \begin{array}{c}\text { Proportion of final } \\ \text { (days) }\end{array} \\ \text { Up to } 7 & (\%) \\ 8-10 & 2 \\ 11-13 & 14 \\ 14 & 80 \\ 15-18 & 92 \\ & 97\end{array}$

Comparison with other media

Viable counts of Mycobacterium tuberculosis $\mathrm{H} 37 \mathrm{Rv}$ were performed in a number of different media. Among those tested were the Kirchner, Fisher and Dubos media with $0 \cdot 125 \%$ agar (in 6 in. $\times \frac{5}{8}$ in. tubes), the same media with $\mathbf{1 . 5} \%$ agar in Petri dishes, Tarshis (1953) blood glycerol medium (without penicillin), Hirsch (1954) charcoal medium with $1 \%$ agar in Petri dishes, and Lowenstein Jensen medium as slopes in screw-capped bottles. Some of the results are shown in Table 4. It is clear that semi-solid Kirchner and Fisher media gave counts as high as any of the standard media tested. In addition, growth in semi-solid Kirchner or Fisher media was easily recognizable earlier than in any of the other media, and viable counts were much easier to read. 
With the solid media in Petri dishes great difficulty was encountered in avoiding contamination or dehydration, especially when cultures were examined at intervals during incubation. These difficulties could be overcome with elaborate precautions but the tubes of semi-solid agar were much easier to handle in large numbers than Petri dishes, cultures could be repeatedly inspected without exposure to risk of contamination and they gave a very low contamination rate when ordinary bacteriological technique was used.

Table 4. Viable counts of Mycobacterium tuberculosis $H 37 R v$ in different media

\begin{tabular}{|c|c|c|c|c|c|}
\hline Medium & $\begin{array}{c}(a) \\
\text { Plates or } \\
\text { tubes } \\
\text { inoculated } \\
\text { (no.) }\end{array}$ & $\begin{array}{c}(b) \\
\text { Plates } \\
\text { spoilt by } \\
\text { contamina- } \\
\text { tion, etc. } \\
\text { (no.) }\end{array}$ & $\begin{array}{c}(c) \\
\text { Successful } \\
\text { plates } \\
\text { (no.) }\end{array}$ & $\begin{array}{c}(d) \\
\text { Colonies } \\
\text { counted } \\
\text { (no.) }\end{array}$ & $\begin{array}{l}(e) \\
\text { Mezn no. of } \\
\text { colonies } \\
(d / c)\end{array}$ \\
\hline Fisher ( $1.5 \%$ agar $)$ & 43 & 14 & 29 & 508 & 17 \\
\hline Kirchner ( $1.5 \%$ agar $)$ & 40 & 8 & 32 & 257 & 8 \\
\hline $\begin{array}{l}\text { Hirsch medium ( } 1 \% \\
\text { agar) }\end{array}$ & 38 & 10 & 28 & 635 & 23 \\
\hline $\begin{array}{l}\text { Tarshis blood } \\
\text { medium (1.5\% agar) }\end{array}$ & 29 & 2 & 27 & 884 & 33 \\
\hline Fisher (0.125 \% agar) & 40 & 1 & 39 & 1117 & 28 \\
\hline $\begin{array}{l}\text { Kirchner }(0 \cdot 125 \% \\
\text { agar) }\end{array}$ & 37 & 1 & 36 & 1164 & 32 \\
\hline dbos (0.125 \% agar) & 30 & $\mathbf{0}$ & 30 & 685 & 23 \\
\hline nstein Jensen & & & 10 & 247 & 25 \\
\hline
\end{tabular}

The growth of other strains of Mycobacterium tuberculosis in semi-solid agar; drug-resistant variants of $H \mathbf{3 7} R v$ strain

Isoniazid-resistant strains. The strain mostly used was the $\mathbf{R} 1$ strain which was resistant to about $10 \mu \mathrm{g}$. isoniazid $/ \mathrm{ml}$. in Dubos liquid medium, showed dependence on haemin in Fisher's medium and was catalase negative (Knox, $1955 b$ ). It grew well and rapidly from large inocula in the standard semi-solid Kirchner agar with serum, though when Dubos cultures of equal age were used for inoculation it grew a little more slowly than the isoniazid-sensitive strain and the final viable counts were usually considerably lower. Comparable inocula of viable organisms grew however at about the same rate with both strains. The resistant strain grew more uniformly and for a greater distance below the surface than the sensitive strain when equally large inocula were used. Like the sensitive strain, it grew poorly or not at all in Kirchner semi-solid agar without serum, but it required at least $10 \%(\mathrm{v} / \mathrm{v})$ serum for optimal growth. Other isoniazid-resistant strains of Mycobacterium tuberculosis var. hominis also grew well in semi-solid Kirchner agar with serum. These included the $\mathbf{R} 4$ variant of the $\mathrm{H} 37 \mathrm{Rv}$ strain resistant to $10 \mu \mathrm{g}$. isoniazid $/ \mathrm{ml}$. and $200 \mu \mathrm{g}$. streptomycin/ $\mathrm{ml}$.; the others were patients' strains which had developed isoniazid resistance during treatment.

Streptomycin-resistant strains. These include the R 3 strain resistant to $200 \mu \mathrm{g}$. streptomycin $/ \mathrm{ml}$., the $\mathbf{R} 4$ strain mentioned above, and several streptomycin-resistant strains isolated from the sputum of patients during treatment. 
These all gave good and rapid growth in the standard semi-solid Kirchner agar with serum.

Mycobacterium tuberculosis var. bovis grew well in Kirchner semi-solid agar with serum.

The B.C.G. strain grew more slowly than the H 37 Rv strain but when $10^{-4}$ dilutions of Dubos cultures were used separate colonies were visible in Kirchner semi-solid agar with serum in 10 days to 2 weeks, and could be accurately counted in 3 weeks or less; this period was considerably shorter than on Lowenstein Jensen medium. Growth was quicker when glycerol was replaced by glucose. The medium was used successfully for viable counts on freezedried cultures of B.C.G.

Sputum concentrates. Specimens of sputum, from patients with pulmonary tuberculosis, homogenized by the alkali method, were inoculated into semisolid Kirchner agar with penicillin or malachite green. Excellent growth was obtained even when the number of organisms was small. The results will be more fully reported elsewhere.

\section{Drug sensitivity tests with cultures of Mycobacterium tuberculosis}

Many drug sensitivity tests were carried out in semi-solid agar media both with cultures of Mycobacterium tuberculosis and with sputum concentrates inoculated directly.

Isoniazid sensitivity tests. These were compared in the liquid media of Kirchner, Fisher and Dubos, and in the same three media with the addition of $0.125 \%$ agar. In all these media the initial end-point with the $\mathbf{H ~} 37 \mathbf{~ R v}$ strain read after 3-4 days of incubation or less was the same, namely, about $0 \cdot 01-0 \cdot 05 \mu \mathrm{g}$. isoniazid $/ \mathrm{ml}$, , but on continued incubation differences began to appear. Of the liquid media, Fisher medium showed the smallest shift in endpoint, Dubos medium showed the steady shift described in an earlier paper (Knox, King \& Woodroffe, 1952), while the Kirchner medium showed a rapid shift within 1-2 weeks, to sometimes as high as $10 \mu \mathrm{g} . / \mathrm{ml}$. Much of this shift is known to be due to inactivation of isoniazid which occurs in uninoculated media and which is more rapid in Kirchner medium than in either of the others, but liquid media gave no information as to the number of organisms involved in this delayed growth. With semi-solid agar medium it was possible to see the number of colonies developing in increasing drug concentrations. Subcultures from such colonies gave inconsistent results when tested for drug sensitivity. Sometimes they showed considerably increased isoniazid resistance; sometimes despite the fact that the colonies had developed in tubes which originally contained high isoniazid concentrations, the subcultures showed apparently normal sensitivity; sometimes they behaved like mixed cultures, showing for example in Dubos medium apparently normal sensitivity when readings were made after 5-7 days of incubation but showing a much more rapid 'shift' in the end-point on longer incubation.

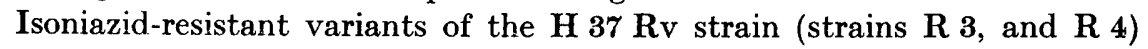
were inhibited by about $10 \mu \mathrm{g}$. isoniazid $/ \mathrm{ml}$. in semi-solid as in liquid media. 
The end-point was sharp and could be read in 3-4 days. It was interesting to note the difference between the isoniazid-sensitive and isoniazid-resistant strains of $\mathrm{H} 37 \mathrm{Rv}$ in Kirchner medium. Both strains eventually grew in $10 \mu \mathrm{g}$. isoniazid/ml., and in the liquid medium the initial end-point of the sensitive strain was difficult to determine, but some growth occurred up to $10 \mu \mathrm{g}$. isoniazid/ml.; in Kirchner semi-solid agar however it could be seen at a glance that only a very small proportion of the organisms in the sensitive strain could grow in $10 \mu \mathrm{g}$. isoniazid $/ \mathrm{ml}$. and that these few organisms were the cause of the rapid shift in end-point which occurred in the liquid medium. On the other hand, it was clear that with the resistant strain all or nearly all the organisms grew in $10 \mu \mathrm{g}$. isoniazid $/ \mathrm{ml}$. and without delay.

Streptomycin sensitivity tests. These were performed in the same media which showed the same sort of differences as they did with isoniazid sensitivity tests. In all of them, however, end-points were sharper and there was less shift on continued incubation than with isoniazid.

p-Aminosalicylic acid $(P A S)$ sensitivity tests. Some preliminary experiments showed that whereas in liquid Dubos medium and liquid Kirchner medium end-points were ill-defined and difficult to read, in semi-solid Dubos agar and particularly in semi-solid Kirchner agar it was easy to see, from the number of colonies which grow, a fairly narrow range of concentrations of PAS within which growth faded from 'growth equal to the control' to a greatly diminished though still large number of colonies only just visible to the naked eye. With such end-points sensitivity tests to PAS can be read like those for isoniazid and streptomycin, in a few days.

In many tests of sensitivity to isoniazid, PAS and streptomycin the effect of inoculum size was investigated. With all three compounds the size of inoculum affected either the initial end-point read after a few days of incubation or the rate of development of resistant colonies, or both. These effects varied with the medium used. The results of these experiments will be more fully reported elsewhere.

Cultures of sputum concentrates. Drug-sensitivity tests performed directly on sputum concentrates gave rapid results with sharp end-points. Many experiments performed to determine the best medium for routine use will be reported separately.

\section{DISCUSSION}

Continued experience of semi-solid media agar has fully confirmed the earlier impression (Knox, 1955a) of their great value for the cultivation of Mycobacterium tuberculosis. A few minor disadvantages will be discussed first. The most obvious criticism is that oxygen may not diffuse freely enough through the depths of the medium to supply the oxygen demands of the growing organisms. At first sight this criticism appears to be supported by the fact that with large inocula growth is much more luxuriant at or near the surface of Kirchner semi-solid agar than in the depths of the medium, where it can be seen that there are numerous minute colonies which develop in the first few days of growth and which then cease to grow any larger, presumably because 
all the oxygen is consumed by the organisms nearer the surface. On the other hand, experiments in which the depth of the column of agar was varied by variation in the size of tube and the volume of medium showed that, in 6 in. $\times \frac{5}{8}$ in. tubes with $2 \mathrm{ml}$. or perhaps even as much as $4 \mathrm{ml}$. of medium, colonies of fairly uniform size developed throughout the medium, at any rate during the early days of incubation, provided the standard technique of inoculation described above was followed, and provided the number of colonies was not greater than a maximum of 40-50. It is clear too that, in the first 2 weeks of incubation, enough oxygen diffuses throughout the medium to give viable counts as high as or higher than the counts given by organisms growing on the surface of several standard solid media where presumably oxygen supply at least in the early days of growth is unlimited. Oxygen in too high a concentration is very inhibitory to $M$. tuberculosis (Moore \& Stenhouse Williams, 1909, 1911); this may indeed partly be the reason for the inhibitory effect of some solid agar media. In semi-solid agar in air it has often been observed that growth of $\boldsymbol{M}$. tuberculosis is better a few $\mathrm{mm}$. below the surface than immediately below it, that this effect increases with decreasing volume of medium, and that it is only when the column of agar (in $6 \mathrm{in} . \times \frac{5}{8}$ in. tubes) is more than $2 \mathrm{in}$. long that there is failure of isolated colonies to develop in the depths of the medium, presumably through lack of oxygen. Further experiments on the effect of oxygen on growth in semi-solid agar will be separately reported. The delayed diffusion of gases which must occur in semi-solid agar is not necessarily altogether disadvantageous for growth of $\boldsymbol{M}$. tuberculosis. One reason why semi-solid agar media sometimes support the growth of small inocula of organisms which fail to grow in the corresponding liquid media may be that essential metabolites, including perhaps $\mathrm{CO}_{2}$, do not diffuse away so rapidly as in liquid media. So far as small inocula of M.tuberculosis are concerned, it seems that oxygen diffuses into semi-solid agar as fast as it is required, at any rate in the early days of incubation and that oxygen lack does not exert any detectable effect upon growth until about the third week of incubation. Oxygen deficiency however is probably one reason for the poor growth which occurs in the depths of media with agar concentrations much above $0.4 \%$.

The number of colonies which can be conveniently counted in semi-solid agar medium is not more than $\mathbf{4 0 - 5 0}$. When the inoculum is diluted in tenfold steps it is sometimes found that a $10^{-5}$ dilution contains too many and a $10^{-6}$ dilution too few colonies. This difficulty is easily avoided, either by using closer dilutions, increasing the number of drops inoculated per tube, or inoculating several duplicates, the number depending on the accuracy required. Sampling of colonies from semi-solid agar for storing or for subcultures is not quite so easy as sampling from the surface of solid media or from liquid media but presents no serious difficulties, except for the preparation of thick suspensions, for which the medium is quite unsuitable.

On the other hand, semi-solid media have certain outstanding advantages. It has been shown that Kirchner semi-solid agar gives very rapid growth of large inocula-growth which is frequently easily recognizable much earlier than in the corresponding liquid medium; it supports the growth of small 
inocula and colonies are visible and countable more easily and earlier than in any other media here used.

Because ordinary 6 in. $\times \frac{5}{8}$ in. test tubes are used it is possible to handle large numbers of cultures at a time, with the use of relatively small volumes of medium and of incubator space. Cultures can be repeatedly examined without in any way exposing them to risk of contamination, contamination rates are very low, and the technique of inoculation is probably much safer to the operator and less liable to be spoiled by contamination than techniques which involve dropping cultures on to the surface of Petri plates exposed to the air.

Because with large inocula growth is easily visible in 2-3 days, the medium is excellent for rapid drug-sensitivity tests. The development of 'resistant' colonies can be followed at intervals during incubation. It is true that these presumptively resistant colonies often showed on subculture lower degrees of resistance than might have been expected from the high concentration of isoniazid initially present in the tubes in which they grew. But the fact that such colonies grow at all in such tubes indicates that even if they are really mixed cultures they do at any rate contain an increased proportion of organisms with resistance greater than normal. With semi-solid agar it is at least possible to obtain a rough estimate of the frequency. with which such 'resistant' organisms occur in a given culture as compared with a normal fully-sensitive strain in the same medium. Thus cultures in semi-solid agar media besides having the advantages associated with the usual serial dilution methods in tubes of liquid media give additional information as to the distribution of resistant organisms in the bacterial population. There are, however, considerable differences between the three media we have used. Fisher's medium perhaps tends to underestimate the proportion of resistant organisms in a predominantly sensitive culture, since growth of resistant strains in Fisher medium is erratic even in the presence of serum, unless haemin is added; Kirchner medium, on the other hand, probably gives an exaggerated picture since the growth of large numbers of colonies in this medium may reflect not so much a general increase of resistant organisms as a more rapid destruction of isoniazid in this medium. Dubos semi-solid agar seems to be the most satisfactory for isoniazid-sensitivity tests, since it is possible to give both an early report on the average sensitivity of a culture and a late report on the proportion of (presumptive) isoniazid resistant organisms.

The Dubos semi-solid agar medium has proved useful for the standardization of cultures of Mycobacterium tuberculosis. It was through its use at the National Collection of Type Cultures for viable counts on freeze-dried cultures of $M$. tuberculosis that our attention was first drawn to the medium. We have used it successfully for assessing the viability of freeze-dried culture of M. tuberculosis $\mathrm{H} 37 \mathrm{Rr}$ and B.C.G. Its possible value for the standardization of B.C.G. vaccine is now being investigated.

Our thanks are due to Mr C. E. Engel, Photographer to the Medical School, Guy's Hospital, for his valuable advice and help in the photography, and to the Medical Research Council for a grant for technical assistance. 

Journal of General Microbiology, Vol. 15. No. 2

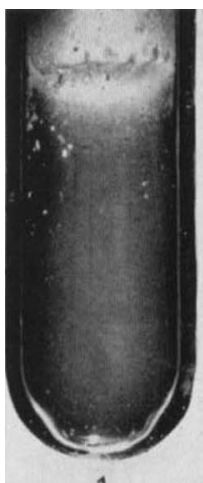

1

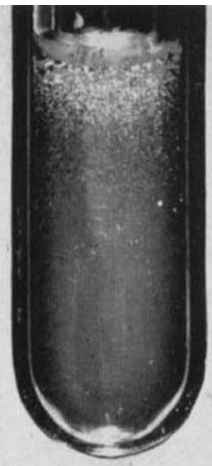

2

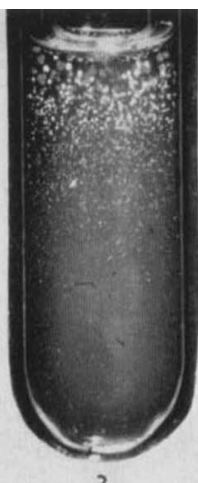

3

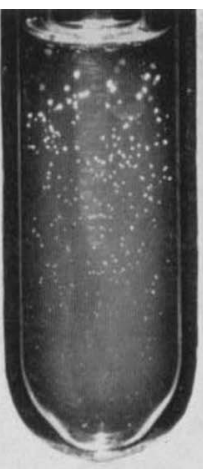

4

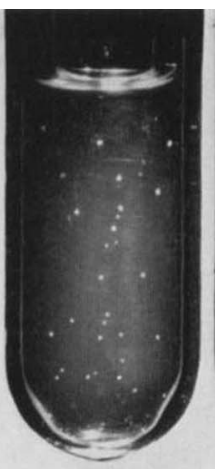

5

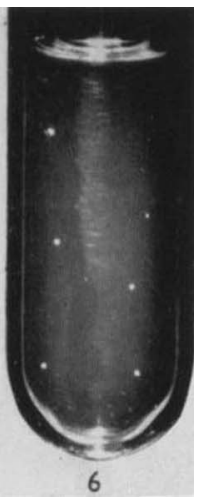

Fig. 1

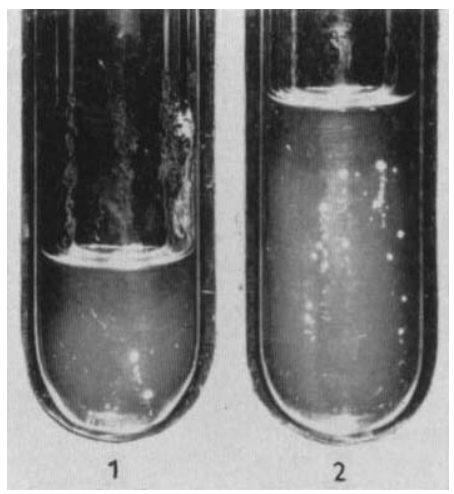

Fig. 2.

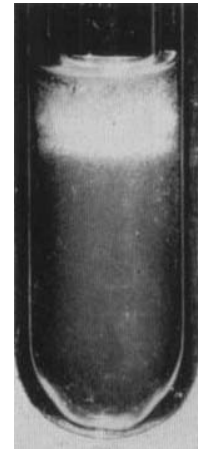

1

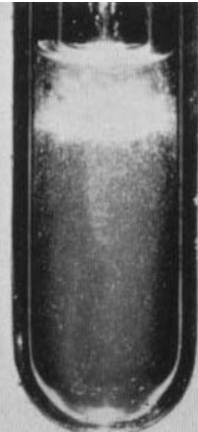

2

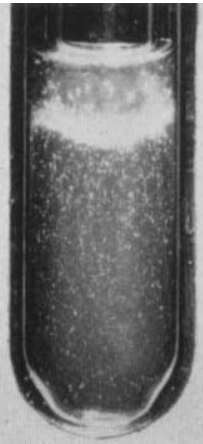

3

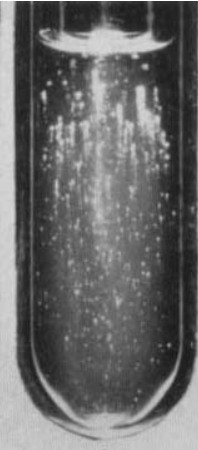

4

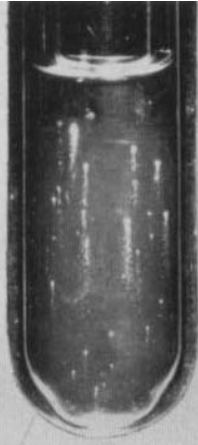

5

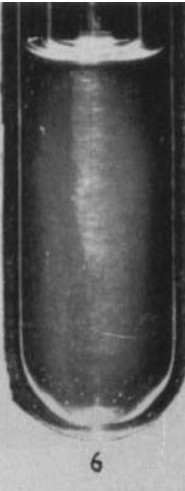

6

Fig. 3

R. KNox, E. SWAit \& R. WOOdROFFe-M. TUberCULOSIS: Growth ON SEMI-SOLID MEDIA

(Facing p. 371) 


\section{REFERENCES}

Dubos, R. J. \& Davis, B. D. (1946). Factors affecting the growth of tubercle bacilli in liquid media. J. exp. Med. 83, 409.

Fisher, M. W. (1952). The altered growth characteristics of isoniazid-resistant tubercle bacilli. Amer. Rev. Tuberc. 66, 626.

Hirsch, J. G. (1954). Charcoal medium for the cultivation of tubercle bacilli. Amer. Rev. Tuberc. 70, 955.

KNox, R. (1955a). Semi-solid agar media for rapid culture of tubercle bacilli. Lancet, ii, 110-112.

KNox, R. (1955b). Haemin and isoniazid resistance of Mycobacterium tuberculosis. J. gen. Microbiol. 12, 191.

KNox, R., KING, M. B. \& Woodroffe, R. C. (1952). In vitro action of isoniazid on Mycobacterium tuberculosis. Lancet, ii, 854.

Mackie, T. J. \& McCantney, J. E. (1953). Handbook of Practical Bacteriology, 9th ed. p. 189. Edinburgh: E and S. Livingstone.

Moore, B. \& Stenhouse Williams, R. (1909). The growth of the Bacillus tuberculosis and other micro-organisms in different percentages of oxygen. Biochem. J. 4, 177.

Moone, B. \& Stenhouse Williams, R. (1911). The growth of various species of bacteria and other micro-organisms in atmospheres enriched with oxygen. Biochem. J. 5, 181.

TARshis, M. S. (1953). Blood media for the cultivation of Mycobacterium tuberculosis. V. Results with agar-agar basal medium and varying concentrations of blood, glycerine and penicillin. Amer. J. clin. Path. 23, 661.

\section{EXPLANATION OF PLATE}

Fig. 1. Viable count of M. tuberculosis $\mathrm{H} 37 \mathrm{Rv}$ in Kirchner semi-solid agar (4 $\mathrm{ml}$. per tube). Incubated 20 days at $37^{\circ}$. Inoculum 0.04 ml. of a 12-day Dubos culture, 1 in 10 dilution in tube 1 and then decimal dilutions to $10^{-6}$.

Fig. 2. Culture of $M$. tuberculosis $\mathbf{H} 37 \mathrm{Rv}$ showing secondary colonies after 28 days incubation at $37^{\circ}$.

Fig. 3. Viable count of $M$. tuberculosis $\mathbf{H} 37 \mathrm{Rv}$ in Dubos semi-solid agar (4 ml. per tube). Incubated at $37^{\circ}$. Inoculum as in Fig. 1. 